\title{
Digital Health in the Management of Pediatric Growth Hormone Therapy - 10 Years of Developments
}

\author{
Ekaterina KOLEDOVA ${ }^{\mathrm{a}, 1}$, Quentin LE MASNE ${ }^{\mathrm{b}}$, Amalia SPATARU ${ }^{\mathrm{c}}$, \\ Merat BAGHA $^{\mathrm{d}}$ and David DIXON ${ }^{\mathrm{a}}$ \\ ${ }^{a}$ Endocrinology Global Medical Affairs, Merck KGaA, Darmstadt, Germany \\ ${ }^{b}$ Connected Health \& Devices, Ares Trading SA, Eysins, Switzerland, \\ an affiliate of Merck KGaA, Darmstadt, Germany \\ ${ }^{c}$ Swiss Data Science Center, ETH Zurich and EPFL, Switzerland \\ ${ }^{d}$ Tiba Medical Inc., Beaverton, OR, USA
}

\begin{abstract}
Growth hormone administration is approved for use in a number of growth failure conditions in children. Optimal growth outcome requires continuous daily injections over many years, leading to problems of persistence and adherence with therapy. The easypod ${ }^{\mathrm{TM}}$ connect ecosystem enables electronic monitoring of injection and dose history, transmitted to a secure cloud database via the internet or cellular networks. Thus, healthcare providers can easily monitor adherence with therapy and be alerted to problems. The growlink ${ }^{\mathrm{TM}}$ patient app has been added to the ecosystem to provide solutions that can engage and educate patients and their families/caregivers. growlink ${ }^{\mathrm{TM}}$ also allows patients to self-report height and weight, enabling healthcare providers to track growth progression. The patient support program, TuiTek and the easypod ${ }^{\mathrm{TM}}$ Augmented Reality (AR) app are being developed within the ecosystem to support telehealth services, increase disease awareness and reduce therapy-related anxiety. easypod ${ }^{\mathrm{TM}}$ connect provides objective assessments of adherence, shown to be maintained at a high level over several years, and analyses showed that increased adherence was significantly associated with a better growth outcome. Studies have identified factors that influence persistence and adherence with GH therapy via the easypod ${ }^{\mathrm{TM}}$ connect ecosystem. These novel technologies are generating solutions that enable datadriven personalized care for children with growth disorders and optimize long-term clinical outcomes.
\end{abstract}

Keywords. Adherence and persistence, childhood growth failure, digital health, easypod $^{\mathrm{TM}}$ connect, growth hormone therapy, patient support

\section{Introduction}

Growth failure in children can be caused by a number of conditions and results in reduced adult height compared with peers and familial potential. Height growth can be improved by therapy with recombinant human 1 growth hormone (r-hGH) for many of these conditions, including GH deficiency, Turner syndrome and lack of catch-up growth after

\footnotetext{
${ }^{1}$ Corresponding Author, Ekaterina Koledova, Endocrinology Global Medical Affairs, Merck KGaA, Frankfurter Str. 250, F135/001, 64293 Darmstadt, Germany; E-mail: ekaterina.koledova@merckgroup.com
} 
being born small for gestational age [1]. Growth response varies between these conditions, but in each case optimal outcome is achieved when GH therapy is started at a young age, before puberty, and is continued over many years, through into adolescence. GH is currently administered most frequently as daily subcutaneous injections for either 6 or 7 days per week. Long-term continuous injections inevitably lead to problems of adherence and persistence over time, which affect outcomes of GH therapy [2]. Barriers to continued adherence and persistence with $\mathrm{GH}$ have been identified [3], and ecosystems incorporating eHealth technologies have been developed to assist in overcoming these, similar to ecosystems in other therapeutic areas such as diabetes and asthma [4-6].

\section{2. easypodTM Connect Development}

$\mathrm{GH}$ administration originally used syringes and needles, but problems occurred due to needle phobia and incorrect dosing, and mechanical pens then auto-injectors were developed to overcome these problems. Auto-injectors evolved to incorporate smart electronics that could collect information on dosing, and the easypod ${ }^{\mathrm{TM}}$ was launched in 2007 and is now approved in more than 40 countries [7]. The development aim was to be easy and friendly to use for parents and children [8,9], and the device now incorporates a skin sensor, automatic needle attachment that hides the needle, audible and visual signals, and customizable injection settings of needle speed and depth to minimize pain [8]. There is a display that shows dose injected, last injection date and time, and remaining dose in the cartridge, along with instructions and reminders available in multiple languages. Regimen settings can be incorporated to allow for schedules of 6 or 7 days of injections per week, and clinics can customize dose settings to enable dose adjustments, as necessary.

The history of doses and timing was initially only available on the display screen, requiring patients to visit the clinic for the healthcare professional (HCP) to be able to see the data. It was quickly recognized that adherence could be better tracked and visualized via computer software, which required connecting the device via a USB port to the docking station. Additional software was soon incorporated into the device, which allows the data on injections, dosing history, and injection settings to be transmitted via the internet using the easypod ${ }^{\mathrm{TM}}$ connect platform. The data is stored in a centralized secure cloud system and can be easily accessed by HCPs, who can then monitor the progress of individual patients and make a more accurate assessment of their requirements. In subsequent years, the ability to transmit data directly from the docking station via cellular networks has been added [5].

\section{The easypod ${ }^{\mathrm{TM}}$ Connect Ecosystem for Remote Monitoring of Therapy}

Patient empowerment is considered a fundamental principle, and it was important to provide tools and solutions that engaged, educated, and complemented the lives of patients and their parents/caregivers during their GH therapy journey. In a digital context, this resulted in the growlink ${ }^{\mathrm{TM}}$ patient app, which was made available in 2017 for use on smartphones. growlink ${ }^{\mathrm{TM}}$ enables patients and their parents/caregivers to obtain information and resources relative to their condition [10]. 


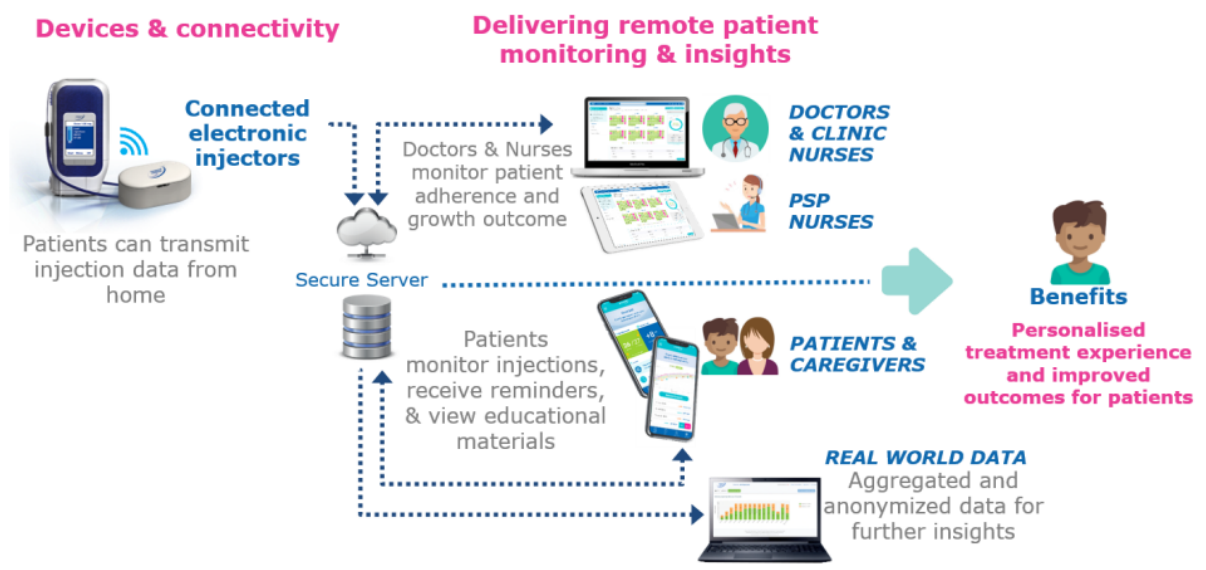

Figure 1. The easypod ${ }^{\mathrm{TM}}$ connect ecosystem for remote monitoring of therapy.

Along with injection reminders, it allows patients to self-report their height and weight data, which is transmitted to the HCP via the easypod ${ }^{\mathrm{TM}}$ connect platform and enables tracking of growth progression. growlink ${ }^{\mathrm{TM}}$ also facilitates interactions between the patients, their healthcare team and patient support services for such things as answering questions or providing supplies such as needles. With increasing uptake of the app, it is anticipated that providing compelling and relevant educational content will mean that the app becomes a routine companion of each patient, driving adherence and persistence with GH therapy to optimize long-term clinical outcomes. Other apps and support solutions that are part of the easypod ${ }^{\mathrm{TM}}$ connect ecosystem are the patient support (behavior change) program known as TuiTek ${ }^{\mathrm{TM}}$ and the easypod ${ }^{\mathrm{TM}}$ augmented reality (AR) app, which are in development and undergoing testing [11]. The aim of these solutions and apps is to support telehealth services, to increase disease awareness and reduce patients' and caregivers' therapy-related anxiety. The TuiTek patient support program enables nurse-led behavioral coaching sessions and eHealth learning to customize the approach to individual caregivers and patients with different needs. The easypod $^{\mathrm{TM}}$ AR app uses augmented reality and 3D animations for interactive lessons, with instructions on correct administration techniques, and engages patients with quizzes and games using an avatar. Overall, the growlink ${ }^{\mathrm{TM}}$ and easypod ${ }^{\mathrm{TM}} \mathrm{AR}$ apps, and the TuiTek patient support program should help to change future management of children with growth disorders by enabling HCPs to address individual issues with adherence, provide eHealth education between clinic visits, and enable patient-centric research to be based on accurate, reliable, aggregated data. They will also support understanding of cultural and healthcare system differences, and variations in technology acceptance between countries.

\section{Testing and Implementing Developments of the easypod ${ }^{\mathrm{TM}}$ Connect Ecosystem}

The easypod ${ }^{\mathrm{TM}}$ connect ecosystem has been assessed using a mixed methods approach to include qualitative and quantitative exploratory research, retrospective analysis of data (after removal of patient identification information) and surveillance studies. Real-world data from thousands of patients in the multi-national 5-year easypod ${ }^{\mathrm{TM}}$ connect 
observational study (ECOS) showed that more than $65 \%$ of patients maintained $\geq 85 \%$ adherence with GH therapy for at least 4 years [12]. ECOS also showed that increased adherence was positively associated with better growth response of the children [13, 14].

More recent work has focused on identifying factors that influence adherence and persistence with therapy and to provide insights on patients likely to show reductions in order to address problems in a timely fashion. Initial analyses comparing patients with long versus short persistence indicate that factors with positive effects on persistence were having $\geq 1$ dose change per year, early age at therapy initiation, high average adherence, customized speed settings of the easypod ${ }^{\mathrm{TM}}$ device, regular follow-up measurements, and being male [15]. Median persistence of use was 3.3 years in patients with all factor values associated with a prolonged persistence of use, while this was 0.6 years in patients with all factor values associated with a low persistence of use. Large differences in persistence were seen between continents. Median persistence of use was $1.0,1.4$, and 2.8 years in the available countries in the Asia-Pacific, America, and Europe regions, respectively. This may reflect socio-economic, cultural, or healthcare system differences, but further analyses are needed [15]. There are provisions within the easypod $^{\mathrm{TM}}$ connect ecosystem for HCPs to enter height and weight information, although this is often not done due to the burden of double-entry. Input of the information into both the health records and the easypod ${ }^{\mathrm{TM}}$ connect system is required; future developments will try to address this issue by increasing connectivity links between the two, but this is difficult due to the complexity of hospital information technologies and rules around implementing such links. Other options include obtaining height measurements at home in an accurate and simple manner and various technologies are being explored that would enable patients to accomplish this via a validated means and provide the information through the growlink ${ }^{\mathrm{TM}}$ app. As more information on growth becomes available within the easypod ${ }^{\mathrm{TM}}$ connect database, there is the possibility to integrate prediction models into the ecosystem and the growlink ${ }^{\mathrm{TM}}$ app. This will provide information, in both tabular and graphical forms, of predicted height over subsequent years and at adult height, for both continued use and if the patient stops use of the easypod $^{\mathrm{TM}}$ device for $\mathrm{GH}$ administration, which could help to influence behavior regarding adherence with therapy.

\section{Conclusions}

This overview of innovative developments that are changing the management of GH therapy for children with growth disorders summarizes the design and implementation of a digital health ecosystem. Patient-centric support means more than just providing a therapy; it also involves enabling all stakeholders to have the tools and information, services needed to achieve the goal of better patient health. This requires an ongoing commitment to improving devices, digital health solutions, and patient support programs, in the easiest, most intuitive, and meaningful way for all involved. Implementation of novel technologies through the easypod ${ }^{\mathrm{TM}}$ connect ecosystem requires meaningful understanding of the needs of patients, and generating solutions that address individual patient circumstances. Such developments will ultimately enable data-driven personalized care for children with growth disorders and optimize long-term clinical outcomes. 


\section{Acknowledgment}

The authors thank Amy Evans of inScience Communications, Springer Healthcare Ltd, UK for providing editorial assistance funded by Merck KGaA, Darmstadt, Germany, in accordance with Good Publication Practice (GPP3) guidelines.

\section{Conflicts of interest}

EK, QLM, and DD are employees of Merck KGaA and EK holds shares in the company. $\mathrm{MB}$ is a former employee of Merck $\mathrm{KGaA}$ and has subsequently provided advisory services to the company.

\section{References}

[1] Navarro R, Dunn JD, Lee PA, Owens GM, Rapaport R. Translating clinical guidelines into practice: the effective and appropriate use of human growth hormone. Am J Manag Care. 2013 Nov;19(15 Suppl):s281-9.

[2] de Arriba Munoz A, Muniz VC, Saez JJA, Beisti A, Llovet E, Aizpun JIL. Impact of adherence on growth response during the first 2 years of growth hormone treatment. Endocrine. 2020. Online ahead of print Dec 7.

[3] Mohseni S, Heydari Z, Qorbani M, Radfar M. Adherence to growth hormone therapy in children and its potential barriers. J Pediatr Endocrinol Metab. 2018 Jan 26;31(1):13-20.

[4] Browne SH, Behzadi Y, Littlewort G. Let visuals tell the story: medication adherence in patients with type II diabetes captured by a novel ingestion sensor platform. JMIR Mhealth Uhealth. 2015 Dec 31;3(4):e108.

[5] Lion F-X. Electronic recording of growth hormone dosing history: the easypod ${ }^{\mathrm{TM}}$ auto-injector. Curr Drug Ther. 2010;5:271-6.

[6] Merchant R, Inamdar R, Henderson K, Barrett M, Su JG, Riley J, et al. Digital health intervention for asthma: patient-reported value and usability. JMIR Mhealth Uhealth. 2018 Jun 4;6(6):e133.

[7] Dahlgren J. Easypod: a new electronic injection device for growth hormone. Expert Rev Med Devices. 2008 May;5(3):297-304.

[8] Bozzola M, Colle M, Halldin-Stenlid M, Larroque S, Zignani M, easypod ${ }^{\mathrm{TM}}$ survey study group. Treatment adherence with the easypod growth hormone electronic auto-injector and patient acceptance: survey results from 824 children and their parents. BMC Endocr Disord. 2011 Feb 4;11:4.

[9] Tauber M, Payen C, Cartault A, Jouret B, Edouard T, Roger D. User trial of easypod ${ }^{\mathrm{TM}}$, an electronic autoinjector for growth hormone. Ann Endocrinol (Paris). 2008 Dec;69(6):511-6.

[10] McNally M, et al. Patients and caregivers perspective on a mobile app that tracks adherence and outcomes in children with growth disorders treated with recombinant human growth hormone (rhGH). Horm Res Pediatr. 2018;90:388.

[11] Malik S, et al. Designing a personalized digital patient support program for patients treated with growth hormone: key design considerations. J Med Internet Res. 2020 Jul 29;22(7):e18157.

[12] Koledova E, Tornincasa V, van Dommelen P. Analysis of real-world data on growth hormone therapy adherence using a connected injection device. BMC Med Inform Decis Mak. 2020 Jul 29;20(1):176.

[13] Koledova E, Stoyanov G, Ovbude L, Davies PSW. Adherence and long-term growth outcomes: results from the easypod ${ }^{\mathrm{TM}}$ connect observational study (ECOS) in paediatric patients with growth disorders. Endocr Connect. 2018 Aug;7(8):914-23.

[14] van Dommelen P, Koledova E, Wit JM. Effect of adherence to growth hormone treatment on 0-2 year catch-up growth in children with growth hormone deficiency. PLoS One. 2018;13(10):e0206009.

[15] Spataru A, et al. Persistence of use in children receiving growth hormone therapy. Abstract accepted for poster presentation at ENDO 2021, March 20-23. 